УДК 512.54

\title{
Analysis of the Stochastic Excitement in a Model of Flow Reactor
}

\author{
Irina A. Bashkirtseva* \\ Polina M. Fominykh ${ }^{\dagger}$ \\ Institute of Mathematics and Computer Science \\ Ural Federal University \\ Lenina, 51, Ekaterinburg, 620000
}

Russia

Received 07.04.2016, received in revised form 17.05.2016, accepted 20.06.2016

A probabilistic mechanism of the stochastic excitement in a model of flow reactor is studied. To analyze this phenomenon in regions of mono- and bistability the stochastic sensitivity function technique and the method of confidence ellipses are used.

Keywords: random disturbances, excitability, stochastic sensitivity, confidence ellipses.

DOI: 10.17516/1997-1397-2016-9-3-269-278.

\section{Introduction}

Mathematical modeling and analysis of nonlinear dynamical systems in the presence of random perturbations attracts attention of researchers from various fields of science. It is well known that noise not only blurs the attractors of deterministic models but it can also lead to qualitative changes in the stochastic dynamics due to nonlinearity of these models. The interplay of nonlinearity and stochasticity results in such phenomena as noise-induced transitions $[1,2]$, stochastic resonance [3,4], noise-induced order [5], chaos [6] and stochastic excitability [7]. These phenomena are found in mechanics, physics, population and neural dynamics. Typically, the system is extremely sensitive to noise near bifurcation points. An analysis of the effects of noise on the oscillatory systems near the soft and hard Hopf bifurcations attracted attention of many researchers (see, e.g., [8-12]).

Mathematical models of chemical kinetics [13] have always been a source of new problems in nonlinear dynamics. The classical example is the model of the Belousov-Zhabotinsky reaction [14]. Among the models of chemical kinetics, models of chemical flow reactor with ideal mixing [15] play an important practical role.

One of the models of chemical flow reactor is considered in this paper. The phenomenon of stochastic excitability is demonstrated and analyzed for this model. There are well developed analytical methods for deterministic models. The analysis of the stochastic dynamics of nonlinear systems faces serious difficulties. Indeed, the probability density function that satisfies the Fokker-Planck-Kolmogorov equation gives a full probabilistic description of stochastic regimes. The direct use of this equation is very difficult even in two-dimensional case. In this situation,

\footnotetext{
*irina.bashkirtseva@urfu.ru

†polinaf93@yandex.ru

(c) Siberian Federal University. All rights reserved
} 
one of the most common methods of stochastic analysis is a direct numerical simulation of random trajectories and their subsequent statistical processing. However, this method is very costly for parametric studies. Then the use of approximations and asymptotics based on the method of quasipotential [16] and the stochastic sensitivity functions technique $[17,18]$ is very actual. The short description of this technique is given in the Appendix.

A brief description of the dynamic regimes of the initial deterministic model of chemical flow reactor is given in Sec. 1.

Probabilistic mechanisms of stochastic excitability in the chemical reactor model with random perturbations are studied in Sec. 2. Sec. 2.1 is devoted to the parametric region where the original deterministic model exhibits at first glance a very simple regime with the stable equilibrium in the form of a single attractor. It is shown that due to the nonlinearity of the model there is intermittency of stochastic fluctuations of large and small amplitudes in this region. Analysis of the probabilistic mechanism of the transition of system to supercritical regime is based on the stochastic sensitivity functions technique and the method of confidence ellipses. The same approach is used in Sec. 2.2 for the study of stochastic excitability in the bistability region.

\section{Deterministic model}

Consider a classical model of chemical flow reactor with ideal mixing introduced by Volter and Salnikov [15]

$$
\dot{x}=-x \exp \left(-\frac{1}{y}\right)+l(a-x), \quad \dot{y}=x \exp \left(-\frac{1}{y}\right)+m(b-y),
$$

where $x$ is the dimensionless current concentration of a reagent, $y$ is the dimensionless temperature, $a$ is the input concentration in the reactor, $b$ is the input temperature and $l$ and $m$ are positive parameters of the reactor. We follow [13] in the paper and use $l=0.5, m=0.25, b=0.165$, $a$ is the control parameter. Even small variations in parameter $a$ can lead to significant changes in the dynamics of the system.

Fig. 1 shows the bifurcation diagram of model (1). One can see two bifurcation points $a_{1}=1.580079$ and $a_{2}=1.582843$. Throughout the whole interval the system has equilibrium. This equilibrium is stable if $a<a_{2}$. If $a>a_{2}$ the equilibrium loses its stability. Along with the equilibrium, the system has another attractor if $a>a_{1}$, namely, a stable limit cycle. It appears at $a=a_{1}$ as a result of the hard Hopf bifurcation. Thus, one can distinguish three intervals that correspond to the qualitatively different dynamic regimes of deterministic system (1). If $a<a_{1}$ the system is monostable with the stable equilibrium in the form of a single attractor. In the interval $a_{1}<a<a_{2}$ the system is bistable with two attractors. They are the stable equilibrium and cycle. Their domains of attraction are separated by a separatrix in the form of the unstable cycle. If $a>a_{2}$ the system is monostable with the limit cycle in the form of a single attractor. In Fig. $1 y$-coordinates of the stable equilibria are shown as a red solid line and unstable equilibria are shown as a red dotted line. Corresponding $y$-coordinates for cycle extreme values are shown blue in color. Solid line indicates stable cycle and dashed line indicates unstable cycle.

Thus, as the input concentration $a$ changes some kind of hysteresis is observed in the reactor. Let us first consider what happens when parameter $a$ increases. If $a<a_{1}$ the reactor goes to the stable regime. When $a$ grows and $a<a_{2}$ minor changes of the equilibrium characteristics of concentration and temperature are observed in the reactor. However, when the parameter passes through the bifurcation point $a_{2}$ where $a>a_{2}$, an abrupt breakdown from equilibrium 


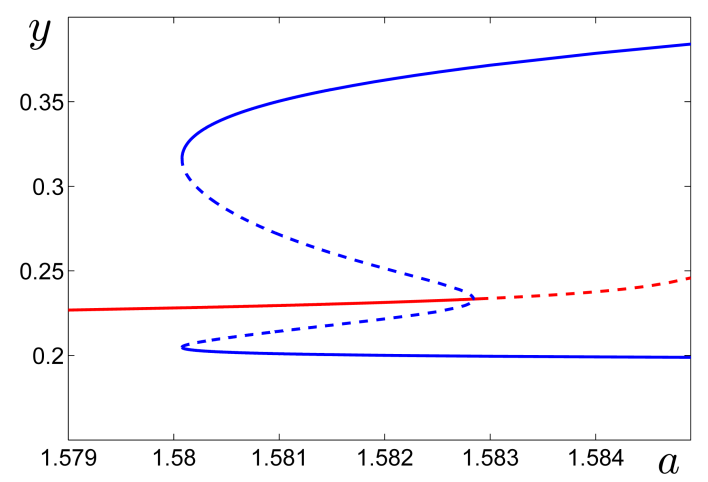

Fig. 1. Bifurcation diagram of deterministic model (1)

regime to oscillatory mode occurs in the reactor. With the decrease of parameter $a$ where $a>a_{1}$ the reactor operates in an oscillatory mode. When parameter $a$ passes through the point of bifurcation $a_{1}$ where $a<a_{1}$ the reverse jump from oscillating mode to equilibrium occurs.

The presence of even small random perturbations can significantly change this scenario.

\section{Dynamics of stochastic model}

Consider model (1) with random disturbances

$$
\dot{x}=-x \exp \left(-\frac{1}{y}\right)+l(a-x)+\varepsilon_{1} \xi_{1}, \quad \dot{y}=x \exp \left(-\frac{1}{y}\right)+m(b-y)+\varepsilon_{1} \xi_{2} .
$$

Here $\xi_{1,2}(t)$ are standard Gaussian noises with parameters $\mathrm{E} \xi_{i}(t)=0, \mathrm{E} \xi_{i}(t) \xi_{i}(\tau)=\delta(t-\tau)$, $\varepsilon_{1,2}$ are noise intensities.

Under the influence of random perturbations stochastic trajectories of system (2) leave the stable equilibrium and randomly oscillate around it. The dispersion of the deviations of these trajectories from the equilibrium depends on the intensity of the noise and stochastic sensitivity of the system. Consider what happens in the system when the noise intensity increases.

\subsection{Stochastic excitability in the region of stable equilibrium}

Consider the dynamics of stochastic system (2) in the region of monostability $a<a_{1}$, where the deterministic model has a stable equilibrium in the form of a single attractor. Here, peculiarities of the phase portrait of deterministic system (1) play an important role. The equilibrium is a stable focus but the behaviour of trajectories depends essentially on the initial deviation. For a small initial deviation, the trajectory monotonically approaches to the equilibrium along the regular spiral curve. If the deviation exceeds some threshold the trajectory at first goes far away from the equilibrium and after that it begins to approach the equilibrium (see Fig. 2 a).

As parameter $a$ approaches the bifurcation point $a_{1}$ this transient phase consists of series of large-amplitude turns (see Fig. 2 b). Initial data that correspond to large-amplitude deviations and damped oscillations are separated by a specific curve (pseudoseparatrix) in the phase plane. This curve separates subcritical and supercritical regions. Computed pseudoseparatrices are shown in Fig. 2 by red dashed lines. 
a)

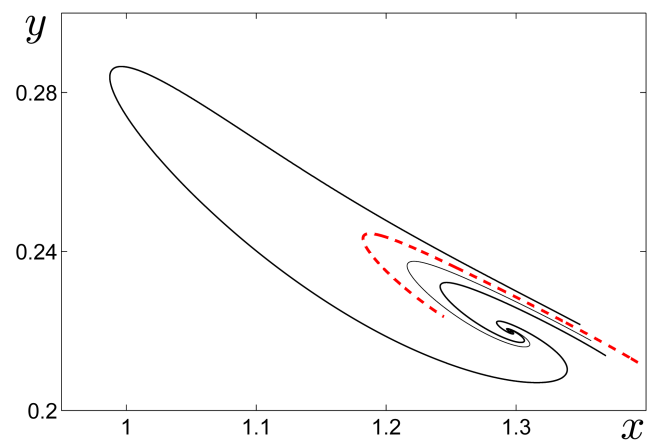

b)

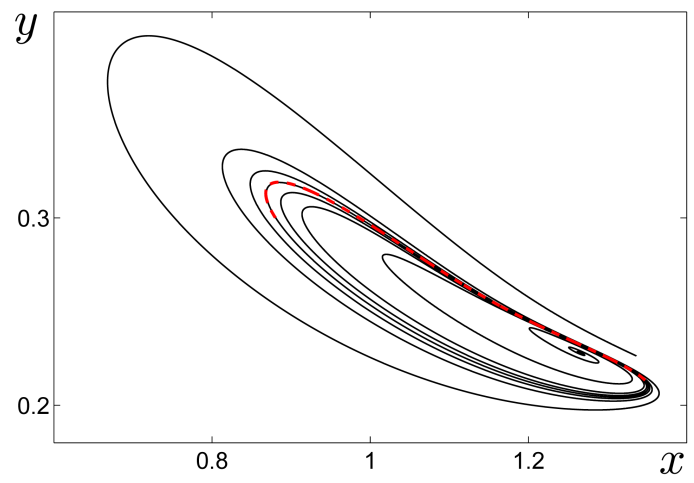

Fig. 2. Phase portraits of the deterministic model for a) $a=1.57$, b) $a=1.58$ (with enlarged fragment)

For small random perturbations stochastic trajectories are concentrated near the stable equilibrium and they are entirely in the subcritical region. The solutions $x(t), y(t)$ of system (2) oscillate with small stochastic amplitudes around the equilibrium values.

When the noise intensity increases stochastic trajectories fall into a supercritical region. They considerably deviate from the equilibrium and return again in the neighborhood of the equilibrium. As a result, stochastic oscillations of small amplitude alternate with stochastic oscillations of large amplitude.

Fig. 3 shows trajectories and corresponding solutions for $a=1.57$ and $a=1.58$ and for various noise intensities $\left(\varepsilon_{1}=\varepsilon_{2}=\varepsilon\right)$. Results of simulation for $a=1.57, \varepsilon=0.0004$ (red) and $\varepsilon=0.0008$ (blue) are shown in Fig. 3 a. As one can see, for $\varepsilon=0.0004$ solutions do not intersect the pseudoseparatrix and the system functions in a subcritical regime. When the noise increases $(\varepsilon=0.0008)$ the trajectory exhibits large-amplitude deviations in crossing the separatrix. The system turns into a supercritical mode where low-amplitude oscillations alternate with large amplitude deviations. These two modes are observed for $a=1.58, \varepsilon=0.0001$ and $\varepsilon=0.0003$ (see Fig. $3 \mathrm{~b}$ ). As one can see, the closer the parameter $a$ to the bifurcation point $a_{1}$ the smaller noise is required to put the system in the supercritical regime. This change clarifies the geometric mechanism of stochastic excitability in the region of the stable equilibrium. It should be noted that stochastic excitability is observed in this model at very low, in fact, at the background noise.

To conduct the parametric analysis of stochastic excitability the stochastic sensitivity function technique and the method of confidence ellipses are used (see Appendix). Eigenvalues $\lambda_{1}(a), \lambda_{2}(a)$ and the corresponding eigenvectors of stochastic sensitivity matrix $W(a)$ represent 
a)
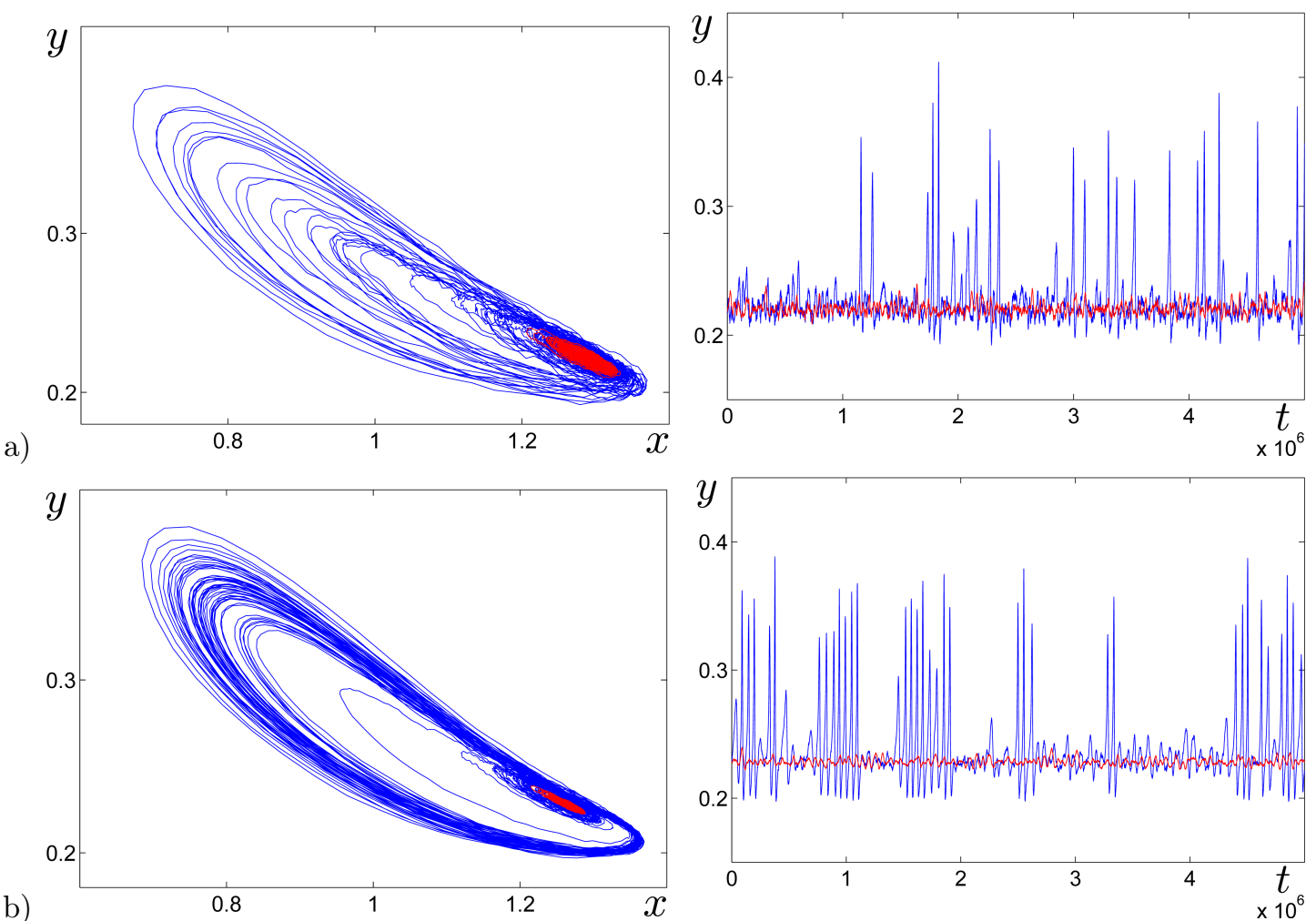

Fig. 3. Phase portraits and solutions $y(t)$ of stochastic system for a) $a=1.57$ with $\varepsilon=0.0004$ (red), $\varepsilon=0.0008$ (blue), b) $a=1.58$ with $\varepsilon=0.0001$ (red), $\varepsilon=0.0003$ (blue)

main spatial characteristics of the probability distribution of random states of stochastic system (2) around the equilibrium of deterministic system (1). The $a$-dependence of $\lambda_{1}(a)>\lambda_{2}(a)$ is ploted in Fig. 4. Large values of the sensitivity $\left(>10^{4}\right)$ are the cause of high excitability of this stochastic model.

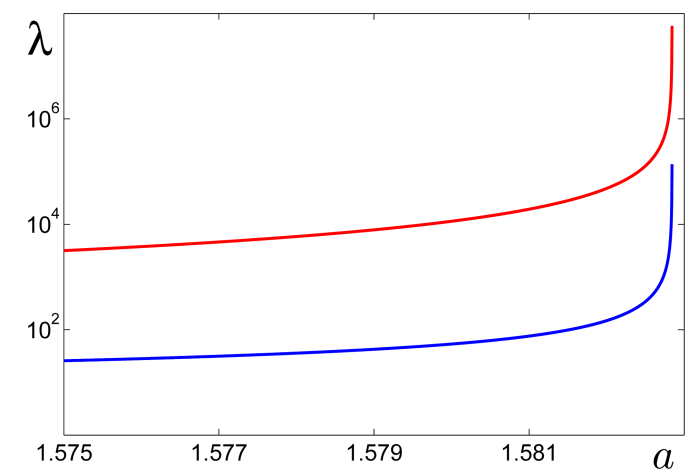

Fig. 4. Stochastic sensitivity of equilibria

The spatial distribution of random states around the equilibrium can be described by confi- 
dence ellipses. Eigenvectors of the stochastic sensitivity matrix $W(a)$ determine the directions of the principal axes and eigenvalues define the size of the semi-axes of the confidence ellipses (see Appendix). Fig. 5 shows random states and the confidence ellipse for system (2) with $a=1.57$, $\varepsilon=0.0001$ and confidence probability $p=0.99$. As one can see, the confidence ellipse adequately reflects the spatial features of the dispersion of random states.

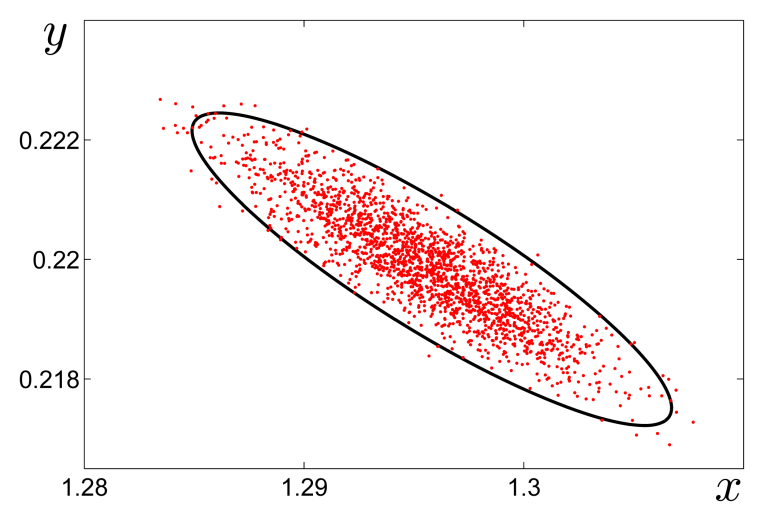

Fig. 5. Random states and confidence ellipse for $a=1.57$ with $\varepsilon=0.0001$

The mutual arrangement of the pseudoseparatrix and confidence ellipses can be used as a criterion of the system transition from the subcritical to the supercritical regime. Indeed, the ellipse size is proportional to the intensity of the noise. For low noise ellipse lies entirely in the subcritical region. The ellipse intersects the separatrix and includes points of the supercritical region with increase in noise. This intersection is taken to be an indicator of the transition to the supercritical regime with large-amplitude oscillations.

Confidence ellipses and pseudoseparatrices of the system for $a=1.57$ and $a=1.58$ and for various noise intensity are shown in Fig. 6 . In Figure 6 a the smaller ellipse corresponds

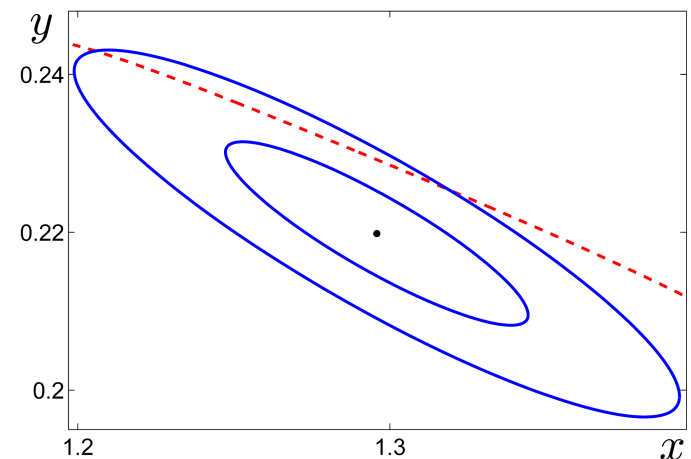

a)

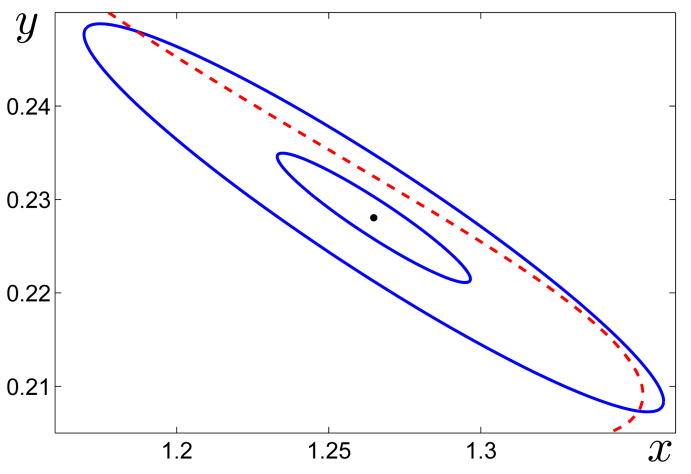

b)

Fig. 6. Confidence ellipses for a) $a=1.57$ and $\varepsilon=0.0004, \varepsilon=0.0008$, b) $a=1.58$ and $\varepsilon=0.0001, \varepsilon=0.0003$. Pseudoseparatrix is shown red in color.

to $\varepsilon=0.0004$ and larger ellipse corresponds to $\varepsilon=0.0008$ for $a=1.57$. As one can see, the ellipse does not touch the pseudoseparatrix for $\varepsilon=0.0004$, so the system operates in subcritical mode (see Fig. $3 \mathrm{a}$, red color). When the noise increases $(\varepsilon=0.0008)$, the ellipse crosses the 
pseudoseparatrix and the system falls into the supercritical mode (see Fig. $3 \mathrm{a}$, blue color). In Fig. $6 \mathrm{~b}$, the smaller ellipse corresponds to $\varepsilon=0.0001$ and the larger ellipse corresponds to $\varepsilon=0.0003$ for $a=1.58$. As one can see, in this case, the transition of the system into the supercritical mode can be also predicted from location of confidence ellipses (see Fig. $3 \mathrm{~b}$ ).

\subsection{Stochastic excitability in a bistability zone}

Let us consider the dynamics of stochastic system (2) in the bistability region $a_{1}<a<a_{2}$. The deterministic model has two attractors in this region (a stable equilibrium and a stable limit cycle) separated by an unstable cycle (see Fig. 7 a for $a=1.582$ and $\varepsilon=0$ ). In this region, the unstable limit cycle plays a role of the separatrix that divides domains of attraction of the equilibrium and the stable cycle.

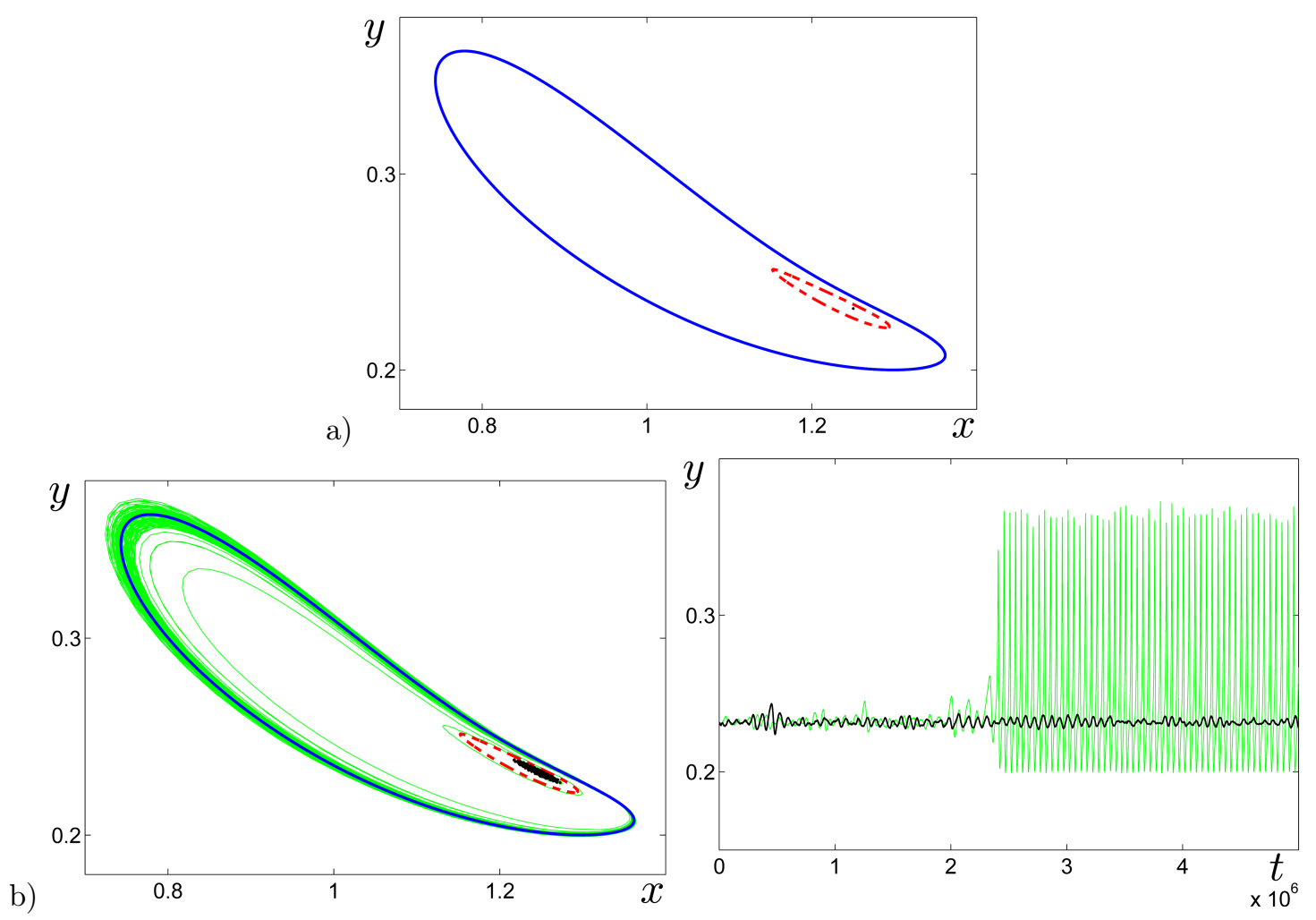

Fig. 7. Generation of large-amplitude stochastic oscillations for $a=1.58$ : a) stable cycle (blue solid line), an unstable cycle (red dashed line) and equilibrium (black dot) of the deterministic system; b) stochastic trajectory and solutions of the stochastic system with $\varepsilon=0.00004$ (black) and $\varepsilon=0.00006$ (green)

Stochastic trajectories that start from a stable equilibrium are located entirely within the domain of attraction of this equilibrium for weak noise. This means that there is a subcritical dynamic regime. The solution $x(t), y(t)$ of system (2) oscillates with a small amplitude around the equilibrium.

When the intensity of noise increases, stochastic trajectories intersect separatrix (unstable limit cycle), go to the domain of attraction of the stable cycle and then continue to move near 
the closed phase curve of this cycle.

Fig. $7 \mathrm{~b}$ shows the trajectory starting from the equilibrium and the corresponding solution for $a=1.582$, the noise intensity $\varepsilon=0.00004$ (black line) and $\varepsilon=0.00006$ (green line). As one can see, for $\varepsilon=0.00004$ random trajectories do not cross the separatrix and the system operates in the subcritical regime. When the noise increases $(\varepsilon=0.00006)$, trajectories intersect the separatrix and start to oscillate in the neighborhood of the stable cycle. Thus, the system turns from small-amplitude oscillations to large-amplitude oscillations.

Using the stochastic sensitivity functions technique and the confidence ellipses method, we obtain the following results (see Fig. 8).

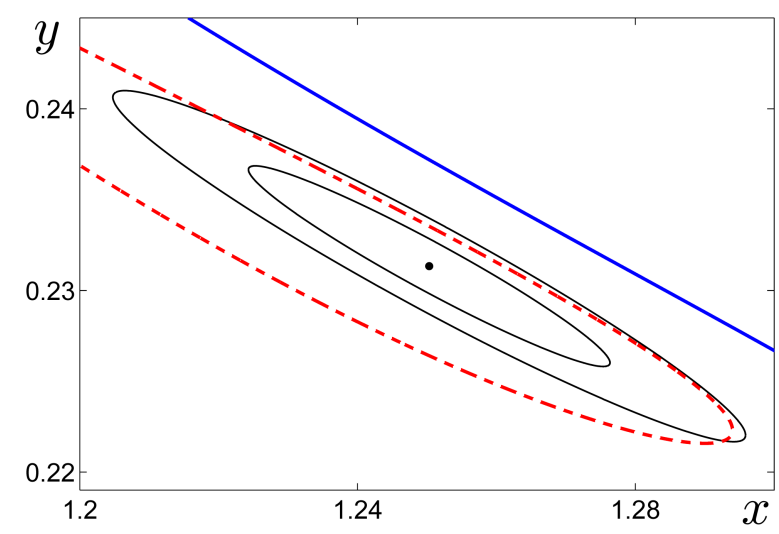

Fig. 8. Confidence ellipses for $a=1.582$ and $\varepsilon=0.00004$ (small ellipse), $\varepsilon=0.00006$ (large ellipse). Unstable cycle is plotted by red dashed line and stable cycle is plotted by blue solid line

As one can see, for $\varepsilon=0.00004$ the ellipse lies entirely in the domain of attraction of the equilibrium. The system operates in subcritical mode (see Fig. 7 b, black line). When the noise increases $(\varepsilon=0.00006)$, the ellipse intersects the separatrix (unstable cycle) and the system falls into a supercritical mode (see Fig.7 b, green line). As can be seen from a comparison with the results of direct numerical simulation of random trajectories, the arrangement of confidence ellipses can be used for the prediction of transition to the supercritical mode.

\section{Appendix}

To study the influence of random perturbations on dynamical systems, the following stochastic system is used

$$
\dot{x}=f(x)+\varepsilon \sigma(x) \xi,
$$

where $x$ is $n$-dimensional vector, $f(x)$ is sufficiently smooth $n$-dimensional vector function, $\varepsilon$ is a scalar parameter of noise intensity, $\sigma(x)$ is a sufficiently smooth $n \times n$-matrix function that defines the dependence of random disturbances on the system states, $\xi(t)$ is $n$-dimensional Gaussian white noise with the parameters $\mathrm{E} \xi(t)=0, \mathrm{E} \xi(t) \xi^{\top}(\tau)=\delta(t-\tau) I, I$ is the identity $n \times n$-matrix.

It is assumed that deterministic system (3) $(\varepsilon=0)$ has an exponentially stable equilibrium $\bar{x}$.

For weak noise one can use the following asymptotics for stationary density $\rho(x, \varepsilon)$ of random states distribution around the equilibrium $\rho(x, \varepsilon) \approx K \cdot \exp \left(-\frac{v(x)}{\varepsilon^{2}}\right)$, where $v(x)$ is the quasipo- 
tential [16]. In a small neighborhood of the equilibrium $\bar{x}$ we use a quadratic approximation for the quasipotential $v(x) \approx \frac{1}{2}\left(x-\bar{x}, W^{-1}(x-\bar{x})\right)$, where $(\cdot, \cdot)$ is the scalar product. This approximation allows us to represent the asymptotic behaviour of the stationary density in the form of a normal distribution $\rho(x, \varepsilon) \approx K \cdot \exp \left(-\frac{\left(x-\bar{x}, W^{-1}(x-\bar{x})\right)}{2 \varepsilon^{2}}\right)$ with covariance matrix $\varepsilon^{2} W$ which characterizes the dispersion of random trajectories of stochastic system (3) around the equilibrium $\bar{x}$.

The matrix $W$ is a unique solution of the algebraic equation

$$
F W+W F^{\top}=-S,
$$

where $F=\frac{\partial f}{\partial x}(\bar{x}), S=\sigma(\bar{x}) \sigma(\bar{x})^{\top}$.

Matrix $W$ relates the noise intensity $\varepsilon^{2}$ to the covariance $\varepsilon^{2} W$ of random states around the $\bar{x}$. It plays a role of the matrix stochastic sensitivity coefficient of the equilibrium $\bar{x}$.

This matrix describes the spatial arrangement of random states of stochastic system (3) around the deterministic equilibrium $\bar{x}$. Using this matrix, we can construct the corresponding confidence domains. In the two-dimensional case, the confidence ellipse is defined by the equation

$$
\left(x-\bar{x}, W^{-1}(x-\bar{x})\right)=2 k^{2} \varepsilon^{2},
$$

Let us assumed that $\lambda_{1}, \lambda_{2}$ are eigenvalues and $u_{1}, u_{2}$ are normalized eigenvectors of $W$. Then using coordinates $z_{1}=\left(x-\bar{x}, u_{1}\right)$ and $z_{2}=\left(x-\bar{x}, u_{2}\right)$, the equation of the confidence ellipse can be written in the standard form $\frac{z_{1}^{2}}{\lambda_{1}}+\frac{z_{2}^{2}}{\lambda_{2}}=2 k^{2} \varepsilon^{2}$.

The stochastic sensitivity functions technique and the method of confidence domains was successfully used in the analysis of noise-induced transitions [17,18] and chaos control [19].

This work was partially supported by the RFBR, no. 16-08-00388 and by the Government of the Russian Federation, Act 211, contract 02.A03.21.0006

\section{References}

[1] W.Horsthemke, R.Lefever, Noise-Induced Transitions, Berlin, Springer, 1984.

[2] Yu.I.Neimark, P.S.Landa, Stochastic and chaotic oscillations, Moscow, Nauka, 1987 (in Russian).

[3] M.D.McDonnell, N.G.Stocks, C.E.M.Pearce, D.Abbott, Stochastic resonance: From Suprathreshold Stochastic Resonance to Stochastic Signal Quantization, Cambridge University Press, 2008.

[4] V.S.Anishchenko, V.V.Astakhov, A.B.Neiman, T.E.Vadivasova, L.Schimansky-Geier, Nonlinear Dynamics of Chaotic and Stochastic Systems. Tutorial and Modern Development, Springer-Verlag, Berlin-Heidelberg, 2007.

[5] K.Matsumoto, I.Tsuda, Noise-induced order, J. Stat. Phys., 33(1983), 87-106.

[6] J.B.Gao, S.K.Hwang, J.M.Liu, When can noise induce chaos? Phys. Rev. Lett., 82(1999), 113-1135. 
[7] B.Lindner, J.Garcia-Ojalvo, A.Neiman, L.Schimansky-Geier, Effects of noise in excitable systems, Physics Reports, 392 (2004), 321-424.

[8] P.S.Landa, Nonlinear Oscillations and Waves in Dynamical Systems, Springer, 1996.

[9] O.V.Ushakov et al, Coherence resonance near a Hopf bifurcation, Phys. Rev. Lett., 95 (2005), 123903.

[10] I.Bashkirtseva, L.Ryashko, H.Schurz, Analysis of noise-induced transitions for Hopf system with additive and multiplicative random disturbances, Chaos, Solitons and Fractals, 39(2009), 72-82.

[11] A.Zakharova, T.Vadivasova et al, Stochastic bifurcations and coherencelike resonance in a self-sustained bistable noisy oscillator, Phys. Rev. E, 81(2010), 011106.

[12] I.Bashkirtseva, T.Ryazanova, L.Ryashko, Stochastic bifurcations caused by multiplicative noise in systems with hard excitement of auto-oscillations, Phys. Rev. E, 92(2015), 042908.

[13] V.I.Bykov, S.B.Tsybenova, Nonlinear models of chemical kinetics, Moscow, Krasand, 2011 (in Russian).

[14] A.M.Zhabotinsky, Concentration Auto-Oscillations, Moscow, Nauka, 1974 (in Russian).

[15] B.V.Volter, I.E.Salnikov, Stability of operation modes of chemical reactors, Moscow, Khimiya, 1981 (in Russian).

[16] M.I.Freidlin, A.D.Wentzell, Random Perturbations of Dynamical Systems, Springer, New York, 1984.

[17] I.Bashkirtseva, L.Ryashko, Sensitivity analysis of stochastic attractors and noise-induced transitions for population model with Allee effect, Chaos, 21(2011), 047514.

[18] I.Bashkirtseva, A.B.Neiman, L.Ryashko, Stochastic sensitivity analysis of the noise-induced excitability in a model of a hair bundle, Phys. Rev. E, 87(2013), 052711.

[19] I.Bashkirtseva, G.Chen, L.Ryashko, Stochastic equilibria control and chaos suppression for 3D systems via stochastic sensitivity synthesis, Communications in Nonlinear Science and Numerical Simulation, 17(2012), 3381-3389.

\section{Анализ стохастической возбудимости в модели проточно- го реактора}

Ирина А. Башкирцева

Полина М. Фоминых

Институт математики и компьютерных наук

Уральский федеральный университет

Ленина, 51, Екатеринбург, 620000

Россия

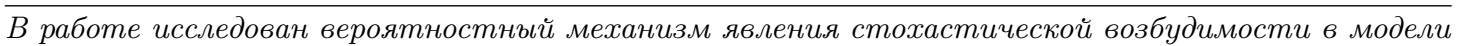
проточного реактора. Для параметрического анализа этого явления в зонах моно- и бистабильности использована техника функиий стохастической чувствительности и метод доверителъных эллипсов.

Ключевые слова: случайные возмущения, возбудимость, стохастическая чувствительность, доверителъные эллипсы. 